\begin{tabular}{|l|l|l|}
\hline & \\
PUCRS & HUMANIDADES & $\begin{array}{l}\text { Educação por escrito, Porto Alegre, v. 12, n. 1, p. 1-8, jan.-dez. } 2021 \\
\text { e-ISSN: 2179-8435 }\end{array}$ \\
\hline http://dx.doi.org/10.15448/2179-8435.2021.1.37396 & \\
\hline
\end{tabular}

SEÇÃO: ARTIGO

\title{
O papel da pesquisa no processo de avaliação das políticas educacionais
}

\author{
The role of research in the educational policy evaluation process
}

\section{Simone Zanatta Guerra ${ }^{1}$ orcid.org/0000-0002-2616-7613 simonezg@hotmail.com}

Renata Cecilia

Estormovski ${ }^{1}$

orcid.org/0000-0001-5714-8928 renataestormovski@gmail.com

Recebido em: 18/03/2020. Aprovado em: 14/09/2020. Publicado em: 23/07/2021.

\section{(c) (1)}

Artigo está licenciado sob forma de uma licença Creative Commons Atribuição 4.0 Internacional.
Resumo: O presente artigo objetiva discutir o papel da pesquisa no processo de avaliação das politicas educacionais, especialmente as avaliações de caráter formativo e plural, das quais, por vezes, as politicas educacionais carecem e que, contraditoriamente, constituem importante estratégia para a leitura das realidades concretas nas quais a politica de educação se materializa. Para isso, busca-se trazer para análise, de maneira breve e não esgotada, elementos para o estudo da pesquisa na área educacional, com destaque às pesquisas qualitativas e, especificamente, à abordagem do ciclo de políticas formulada por Stephen Ball e colaboradores. A pesquisa define-se como qualitativa, exploratória e bibliográfica e inclui entre os referenciais teóricos que a embasam André, Arretche e Mainardes. Aponta-se, nessa construção, o ciclo de politicas como uma possibilidade de realizar avaliações de políticas educacionais engajadas socialmente e comprometidas com a investigação dos impactos das desigualdades educacionais e sociais.

Palavras-chave: Avaliação de politicas públicas. Pesquisa em educação. Politicas Educacionais.

Abstract: This article aims to discuss the role of research in the process of evaluating educational policies, especially evaluations of a formative and plural character, which educational policies sometimes lack and which, contradictorily, constitute an important strategy for reading concrete realities in which the education policy materializes. To this end, we seek to bring, for a brief and not exhausted analysis, elements for the study of research in the educational area, with emphasis on qualitative research and, specifically, the approach of the Policy Cycle formulated by Stephen Ball and collaborators. The research is defined as qualitative, exploratory and bibliographic, and includes among the theoretical references that support Andre (2001), Arretche (2013) and Mainardes (2006). In this construction, the Policy Cycle is pointed out as a possibility to carry out evaluations of educational policies that are socially engaged and committed to investigating the impacts of educational and social inequalities.

Keywords: Evaluation of public policies. Education research. Educational Policies.

\section{Introdução}

As políticas públicas traduzem-se em ações desenvolvidas pelo Estado no atendimento às demandas da sociedade, a exemplo das políticas de assistência social, saúde e educação, sendo estas últimas também denominadas de politicas educacionais. Como as demais, tais politicas estão ancoradas em bases ideológicas e em intencionalidades, que repercutem nos efeitos atingidos na e pela prática pedagógica. Nesse processo, torna-se importante o desenvolvimento de formas de avaliação dessas políticas que se deem de maneira formativa, reconhecendo as 
multiplicidades das realidades concretas em que as ações estatais se materializam. Essa avaliação se mostra relevante para se pensar o impacto de determinada política e/ou a elaboração de outras políticas. Desse modo, uma das estratégias possiveis é a pesquisa em educação, considerando que as políticas educacionais constituem importante campo de estudo dessa disciplina.

Como este trabalho busca trazer a perspectiva de uma avaliação que é formativa, critica e plural, também é importante observar que a teoria desempenha um papel de extrema relevância nesse processo de pesquisa - que aparece aqui como uma possibilidade de avaliação das políticas educacionais -, pois, como afirmam Fávero e Tonieto (2016, p. 35), fundamentando-se em Ball:

A falta de teoria limita o trabalho do pesquisador, já que a leitura de mundo deste fica restrita a preconcepções ingênuas, tanto ontológica quanto epistemologicamente, o que compromete de forma significativa a produção do conhecimento científico no campo educacional.

Os pesquisadores também ressaltam, com base em Popper, que "é a teoria que nos auxilia a projetar o que fazer, como fazer, para onde ir, onde ter um interesse, o que observar, o que fazer com o percebido" (FÁVERO \& TONIETO, 2016, p. 41-42).

Desse modo, partindo do pressuposto de que "o propósito da teoria é desfamiliarizar práticas e categorias vigentes para fazê-las parecer menos evidentes e necessárias, abrindo espaços para a invenção de novas formas de experiência" (BALL, 2011, p. 93), o objetivo do presente trabalho é discutir o papel da pesquisa - cuja base teórica deve lhe dar a necessária sustentação - no processo de avaliação das políticas educacionais. Constrói-se, para isso, um breve levantamento de elementos desse campo de estudos na área educacional, destacando-se a abordagem do ciclo de políticas, formulada por Stephen Ball e colaboradores, como Richard Bowe.

Ressalta-se que o problema que move esta análise é "qual o papel da pesquisa na avaliação de politicas educacionais?" e que este trabalho é resultado de uma pesquisa qualitativa, exploratória e bibliográfica, que inclui entre os referenciais teóricos que a embasam André (2001), Arretche (2013), Mainardes (2006) e Santos (2008). O texto está estruturado em três partes. Na primeira, serão discutidas diferentes concepções de avaliação das políticas educacionais, apontando-se a importância da consideração de aspectos qualitativos em seu desenvolvimento. A segunda parte tratará das especificidades da pesquisa em educação, considerando-se o contexto atual de produção de conhecimento. Na última seção, a abordagem do ciclo de políticas será apontada como uma das possibilidades de realização de pesquisa na área educacional e de qualificação dos processos avaliativos.

\section{Avaliação das políticas educacionais: da reflexão crítica à tomada de decisões}

Ao se empreender uma discussão em que se busca refletir sobre a avaliação das políticas educacionais, convém compreender esse movimento como pertencente a um encadeamento de etapas que resultam na materialização das ações do Estado, como já brevemente explicitado nesta escrita. Nesse sentido, Jaime et al. (2013) discutem que o campo de estudos denominado de políticas públicas, pensado em sua gênese pelo americano Harold Lasswell, buscava inicialmente uma articulação entre o conhecimento científico e as tomadas de decisão. Nessa construção, desenvolveram-se duas dimensões, sendo uma pautada no conhecimento do processo da política e outra interessada em utilizar esses saberes para qualificar o campo de estudos das políticas públicas. Assim, vinculadas à primeira dimensão, surgem pesquisas que se dedicam a pensar aquilo que é nomeado na literatura da área como modelo sequencial, processual, de fases ou de etapas de politicas, em que se situa, como exemplo, o ciclo de políticas de Ball e Bowe, a ser abordado posteriormente neste estudo.

Dessa forma, salienta-se como basilar compreender, a priori, que as políticas públicas se estruturam mediante processos que envolvem distintas etapas articuladas - e não necessariamente sucessivas -, entre as quais se situa o momento dedicado a pensar sobre os resultados de uma ação por meio 
de uma avaliação. Arretche (2013) afirma que essa etapa, por se estabelecer como uma atribuição de valor, é construida por meio de principios, explícitos ou não, e não pode ser concebida de forma neutra ou técnica. Para a autora, a avaliação de políticas públicas implica, necessariamente, o uso de instrumentos de análise que tornem menos tênues os limites entre as opiniões de quem avalia e os resultados expressos de uma política.

Nessa concepção, Arretche (2013) aponta três tendências de avaliação de políticas públicas retratadas na literatura desse campo: a que analisa a efetividade, outra que se preocupa com a eficácia e aquela que se dedica a pensar a eficiência. $O$ primeiro modelo avalia uma política pelo impacto, que pode ser vinculado unicamente a ela, nas condições sociais de vida de seus usuários com base em conceitos de justiça social e de igualdade. De acordo com a autora, há uma carência de avaliações embasadas nesse modelo no cenário brasileiro, considerando-se a dificuldade de definir os resultados de uma política nesses termos. Por conseguinte, geralmente se usa esse fator - de impacto nas condições sociais de vida - como um dos pontos de análise nos estudos desenvolvidos com base em outros modelos. Na sequência, Arretche apresenta a segunda tendência, da eficácia, como sendo a mais comum em pesquisas desse gênero. Esse modelo avalia quais metas e instrumentos apontados no desenho de uma politica foram efetivamente conquistados e relaciona, portanto, objetivos a resultados. Já o terceiro formato, da eficiência, considera o esforço em se concretizar dado plano vinculado ao impacto alcançado, pensando nos recursos públicos investidos para que certa política alcançasse determinados efeitos.

Esses formatos, contudo, são tratados por Arretche (2013) como modelos possiveis de avaliação, sendo apenas pontos considerados para a ampliação, retificação ou supressão de uma política, já que a opinião pública - e sua pressão no jogo político - tende a se sobressair nesse processo.
Tais abordagens, nesse sentido, se consolidam como fontes que qualificam os juizos de valor da população quanto às ações do Estado, o que reafirma a importância de se desenvolverem estudos comprometidos com a verificação das potencialidades das politicas como formas de melhorarem as condições de vida e a formação, no caso das politicas educacionais, dos sujeitos.

Todavia, torna-se visivel que as avaliações de politicas mais disseminadas no contexto atual, principalmente nos meios de comunicação de massa, são as quantitativas, que consideram interesses economicistas vinculados à racionalização de recursos, na mesma medida em que, muitas vezes, desconsideram importantes fatores qualitativos, prejudicando o caráter reflexivo, plural e formativo do processo avaliativo. No campo educativo, por exemplo, o Índice de Desenvolvimento da Educação Básica² é o principal indicador avaliativo utilizado para retratar a qualidade das escolas brasileiras e, como consequência, das políticas educacionais. Para elaborá-lo, utilizam-se testes de desempenho aplicados em algumas séries do ensino fundamental ( $5^{\circ}$ e $9^{\circ}$ anos), que envolvem apenas duas disciplinas (língua portuguesa e matemática) e que são relacionados aos indices de aprovação escolar. Nesse modelo de avaliação, definido como de larga escala, as peculiaridades dos contextos educativos, as diferenças existentes entre as escolas e o próprio capital cultural $^{3}$ dos discentes são desconsiderados, em um modelo de avaliação que prioriza resultados numéricos, sem considerar questões de pluralidade, de participação ou mesmo os avanços individuais dos estudantes.

Ante o exposto, julga-se necessário pensar em possibilidades e estratégias de avaliar as politicas públicas considerando-as em sua dimensão qualitativa, pensando nas particularidades dos contextos de implementação e em seus efeitos reais na vida dos usuários, desvinculando-se de padrões de mercado estimulados continuamente por organismos internacionais. Retomando as con-

Ver mais em: http://portal.inep.gov.br/ideb

3 O conceito de capital cultural é resultante de estudos realizados inicialmente por Pierre Bourdieu para apontar que os diferentes resultados educacionais alcançados pelos alunos não dependiam apenas de seus esforços ou de caracteristicas individuais, mas das distintas condições sociais em que viviam. Sobre tal aspecto, ver mais em Nogueira e Nogueira (2002). 
siderações de Arretche (2013), é necessário que se conceba o processo de avaliação das políticas públicas, das quais as educacionais são parte, com instrumentos que a tornem científica sem desconsiderar sua subjetividade, buscando maneiras de retratar seus impactos para os cidadãos.

Por esse ângulo, a pesquisa em educação apresenta-se como uma possibilidade para que se empreenda um modelo de avaliação das políticas educacionais pautado nos principios de formação, reflexividade e pluralidade, considerando-se os aspectos qualitativos necessários para apreender a completude e a complexidade dessas políticas. Assim, a próxima seção deste estudo abordará, brevemente, elementos da pesquisa em educação no contexto atual, associando-os à avaliação de políticas educacionais.

\section{Pesquisa em educação: ferramenta para avaliar as políticas educacionais}

Ao pautar a pesquisa em educação como uma possibilidade de avaliação reflexiva e crítica das políticas educacionais, é necessário, em um primeiro momento, destacar a qual pesquisa esta escrita se refere. Isso porque, assim como há múltiplos modelos de avaliação, as formas de se fazer pesquisa - suas epistemologias, métodos e procedimentos - também são diversas. Nesse sentido, Santos (2008) traz alguns elementos para se pensar a especificidade da pesquisa nas ciências humanas. Defendendo uma posição epistemológica antipositivista, o sociólogo propõe que o conhecimento científico é socialmente construido e, portanto, não é neutro. Denunciando a crise do paradigma dominante, ou seja, do modelo global de racionalidade científica da modernidade, no qual as ciências humanas muitas vezes meramente apanhavam para si o modelo de pesquisa das ciências naturais e exatas - levando à ideia do "mundo-máquina" determinista -, Santos aponta para a emergência de um novo paradigma, denominado por ele de paradigma emergente.

Nesse contexto, o paradigma emergente adentra no terreno da reflexividade e do questionamento às certezas absolutas. Ante essa trans- formação, "a análise das condições sociais, dos contextos culturais, dos modelos organizacionais da investigação científica, antes acantonada no campo separado e estanque da sociologia da ciência, passou a ocupar papel de relevo na reflexão epistemológica" (SANTOS, 2008, p. 50-51). Buscando escapar das amarras do mecanicismo e de simplificações arbitrárias da realidade social, de acordo com Santos (2008), o novo paradigma abriria, por meio da reflexão crítica, espaço para uma ciência que se preocupa com questões qualitativas, tendo em vista que essas se apresentam limitadas no paradigma dominante, cujo foco tende a ser as questões de cunho quantitativo.

Para Santos (2008), a ação humana é profundamente subjetiva, o que impõe critérios epistemológicos particulares que, costumeiramente na trajetória das pesquisas sociais, foram desconsiderados por relações de poder institucionalizadas e até pela industrialização da ciência. Em um processo de omissão quanto aos contextos singulares e às realidades de vida dos sujeitos, que foram reduzidos à métrica de estudos valorados numericamente, nessa construção, as pesquisas voltadas às humanidades tornaram-se desprovidas de seu sentido social. Dessa forma, o autor propõe um "paradigma de um conhecimento prudente para uma vida decente" (SANTOS, 2008, p. 60), que se daria pela união entre o paradigma científico e o paradigma social, afirmando que 1) todo o conhecimento científico-natural é científico-social; 2) todo o conhecimento é local e total; 3) todo o conhecimento é autoconhecimento; e 4) todo o conhecimento científico visa constituir-se senso comum.

Com base nisso, tem-se a perspectiva de que as ciências humanas, campo no qual se inserem as pesquisas em políticas educacionais e demais políticas públicas, não devem buscar a mecanização do mundo e das relações sociais sob a premissa de garantia do rigor científico. Desmistifica-se, assim, a existência de verdades absolutas e compreende-se que o conhecimento científico, quando socialmente construido, não é neutro, o que impõe a utilização de instrumentos próprios para sua análise. Nesse sentido, Santos (2008, p. 73-74) destaca a importância da con- 
textualização da pesquisa, na medida em que "o conhecimento é tanto mais rigoroso quanto mais restrito é o objeto sobre qual incide".

Logo, em uma conjuntura em que há questionamentos em relação às verdades absolutas, com a crise do paradigma dominante e a ascensão de novas formas de se fazer pesquisa em ciências humanas, André (2001) traz contribuições pensando na garantia do rigor científico e da qualidade na pesquisa em educação. A pesquisadora afirma que a produção e a análise do que é produzido na área educacional é um processo contínuo e coletivo, destacando a importância da promoção do debate em diversos espaços, como universidades, escolas e agências de fomento, para assegurar a qualidade da pesquisa em educação.

Além disso André (2001), ao traçar o percurso dos novos rumos da pesquisa em educação no contexto atual, refere que se passou a recorrer a enfoques multi e transdisciplinares para possibilitar a compreensão e interpretação de questões ligadas à área educacional. Quanto aos aspectos metodológicos, afirma que houve uma ampliação nos estudos de cunho qualitativo. A autora cita que, nesse contexto, a construção de critérios de validação do rigor científico das pesquisas na área "é uma tarefa coletiva e de longo prazo" (2001, p. 58), todavia, já nos traz, a priori, alguns elementos importantes, por exemplo, a relevância científica e social da pesquisa, o objeto bem definido, a adequação da metodologia e a fundamentação da análise.

Ratificando tais percepções, Sandín Esteban (2010) aprofunda as reflexões acerca do rigor científico em pesquisas qualitativas, próprias das humanidades. A autora afirma que os critérios de validação têm sido repensados, considerando-se o reconhecimento de que a subjetividade do pesquisador e dos sujeitos com quem um estudo dialoga, os métodos e até o tempo destinado à coleta e à análise dos dados (SANTOS GUERRA, 1990, apud SANDÍN ESTEBAN, 2010) tornam-se fluidos e impactam a reconstrução da realidade. Assim problematiza o conceito de validade, usualmente utilizado em pesquisas quantitativas, reformulando-o ante aquelas qualitativas, em que a interpretação do pesquisador é enfatizada e não há possibilidade de se estabelecerem regras únicas.

Dessa forma, concebendo a construção do conhecimento em uma perspectiva social, Sandin Esteban (2010) aponta que a dicotomia entre uma busca objetiva pela verdade universal e uma definição quanto a um método específico para eliminar as subjetividades de uma pesquisa questões fundamentais à cientificidade - precisa ser superada pela articulação entre o objetivo e o subjetivo (EISNER, 1998 apud, SANDÍN ESTEBAN, 2010). Para a autora, as experiências vividas pelos sujeitos são processos, trocas, "transações" (termo usado por Eisner), que não podem ser encaixados em padrões de pura objetividade ou subjetividade.

Com base no exposto, percebe-se que a pesquisa em ciências humanas, e mais especificamente na área educacional, tem passado por mudanças no contexto atual. Essas mudanças fomentaram e fomentam a reflexão crítica, a contextualização da pesquisa em dada realidade concreta e a construção de instrumentos qualitativos de pesquisa, sem, importante ressaltar, perder a qualidade científica da investigação. É por esse contexto que se acredita no potencial da pesquisa em educação para desenvolver possibilidades outras de avaliação das políticas educacionais, de modo que considere as questões de cunho qualitativo, como a realidade em que tais políticas se materializam e a pluralidade de contextos sociais. Ressalta-se que a abordagem do ciclo de políticas é considerada, nesta escrita, como relevante estratégia para realizar uma avalição de politicas educacionais pautada nas concepções de pesquisa expostas.

\section{A abordagem do ciclo de políticas: da influência à estratégia política}

No contexto atual, em que se discute amplo leque de estratégias para o desenvolvimento de pesquisas em educação, a abordagem do ciclo de políticas aparece como uma das possibilidades de compreensão, de forma critica e aprofundada, acerca das politicas educacionais e de seus processos avaliativos. Por conta disso, e por considerá-la potente em seus objetivos, 
esta escrita traz alguns elementos para o entendimento dessa abordagem.

O ciclo de políticas, que, conforme Mainardes et al. (2011), pode ser considerado um método de pesquisa de políticas, foi proposto pelo sociólogo Stephen J. Ball e por colaboradores, como Richard Bowe. Nessa abordagem, "o processo de formulação de políticas é considerado como um ciclo contínuo, no qual as políticas são formuladas e recriadas" (MAINARDES et al., 2011, p. 157). Essa estratégia é utilizada em diversos paises como uma referência na análise da trajetória de políticas sociais e educacionais por meio de cinco contextos que se relacionam entre si sem dimensão temporal ou sequencial. Ou seja, não se evidenciam como etapas lineares (MAINARDES, 2006), mas como fases que se conectam e interferem umas nas outras.

Inicialmente, Ball e Bowe propuseram que as políticas fossem analisadas por meio de três contextos: o de influência, o de produção de texto e o da prática. Todavia, em 1994, Ball acrescentou à discussão mais dois contextos: o de resultados/ efeitos e o de estratégia política. Ressalta-se que, não se constituindo etapas lineares, como já indicado, esses cinco contextos não devem ser analisados de forma isolada, mas sim cada um em estreita relação com os demais (MAINARDES, 2006; MAINARDES et al., 2011). Cabe destacar ainda que, com essa construção, acrescentou-se aos estudos de políticas, especialmente das educacionais, a necessidade de se considerarem aspectos da micropolítica, como as ações dos profissionais que atuam em niveis locais, em uma relação entre micro e macrocontextos (MAINARDES, 2006). Dessa maneira, qualificaram-se as discussões, denotando a complexidade, a dinamicidade e os movimentos muitas vezes controversos de uma decisão pública.

Faz-se importante construir uma breve introdução a cada contexto, de modo que possibilite a compreensão da abordagem do ciclo de políticas e da sua completude. Conforme Mainardes (2006), o primeiro contexto - contexto de influência - situa-se na arena de disputa de interesses, na qual diferentes grupos concorrem para influenciar a definição das políticas educa- cionais, ou seja, quais suas intencionalidades e seus fins. Nessa arena, localizam-se partidos políticos, governo, poder legislativo, meios de comunicação social, comissões, grupos representativos e agências multilaterais. Esse contexto está intrinsecamente relacionado com o segundo - contexto de produção de texto -, que, traduzindo-se no resultado dessas disputas e acordos, é onde os textos políticos são construídos. Por textos políticos, entendem-se legislações, pronunciamentos oficiais, videos, entre outras representações da política.

Já o terceiro contexto - contexto de prática "[...] é onde a política está sujeita à interpretação e recriação e onde a política produz efeitos e consequências que podem representar mudanças e transformações significativas na política original" (MAINARDES, 2006, p. 53). Dessa maneira, essa abordagem parte da concepção de que "[...] as políticas não são simplesmente 'implementadas' dentro dessa arena (contexto da prática), mas estão sujeitas à interpretação e, então, a serem 'recriadas'" (MAINARDES, 2006, p. 53). Por conta disso, esse método pressupõe que os profissionais da educação desempenham papel ativo no processo de interpretação e recriação.

O quarto contexto é o de resultados/efeitos. Esse contexto se preocupa fundamentalmente com as questões relacionadas à justiça, à igualdade e à liberdade individual. As políticas devem, com base nele, ser analisadas em suas interações com as desigualdades existentes, observando-se seu impacto na relação com estas últimas. Já o quinto contexto é o contexto de estratégia política, que busca a identificação de atividades sociais e políticas que seriam necessárias para lidar com as desigualdades que foram construidas e/ou reproduzidas por determinada política que fora objeto de estudo da pesquisa (MAINARDES, 2006).

Pela breve exposição dos cinco contextos propostos nessa abordagem, é possivel perceber a maneira orgânica com que eles se articulam, buscando possibilitar uma compreensão crítica das políticas educacionais - suas intencionalidades, interesses, modos de funcionamento, relação com o contexto social, entre outros aspectos relevantes. 
Por conta disso, esse método tem trazido grandes contribuições para se pensar as politicas de educação, bem como as demais políticas sociais.

Ainda é relevante salientar, conforme já indicou Mainardes (2006), que esse método de pesquisa abre possibilidade para utilizar diversos procedimentos para gerar dados, na medida em que cada contexto e/ou cada política objeto de análise pode ser mais bem compreendido por meio de diferentes estratégias, a exemplo da pesquisa bibliográfica, da pesquisa documental, da entrevista, da etnografia, entre outras. Por fim, novamente ressalta-se a importância da pesquisa, independentemente de qual for a metodologia adotada, estar ancorada em uma teoria que permita ao pesquisador a reflexividade crítica, como já afirmava Ball (2011).

Nessa construção, também se salienta a compreensão de que a forma como uma política é entendida está em relação com a maneira pela qual pode ser avaliada, e o ciclo de políticas evidencia modos distintos de se pensar nos resultados de uma ação estatal materializados na vida e nas realidades em que se inserem os sujeitos. O potencial dessa abordagem em problematizar e valorar o contexto em que se concretizam os efeitos de uma política traz possibilidades para uma avaliação que, contraditoriamente ao padrão imposto por avaliações externas, como citado, contribua para construir relatos qualitativos que descrevam os impactos reais de uma política na superação de desigualdades sociais e, entre elas, educacionais.

Além disso, o entendimento dessa abordagem de que suas etapas constituem atravessamentos não lineares corrobora a percepção de que aquilo que foi sentido pelos usuários de uma politica deve ser utilizado para reelaborá-la ou para construir outros instrumentos de ação política que amplifiquem o alcance de um objetivo. Assim, em uma construção dinâmica e reflexiva, torna-se possivel repensar as concepções, os métodos e os formatos dos processos avaliativos das políticas educacionais por meio de um olhar ancorado em elementos da pesquisa desse campo e também nas contribuições de Ball e Bowe.

\section{Considerações finais}

As políticas educacionais, como parte das políticas sociais, estabelecem-se como direitos conquistados historicamente pelos sujeitos, em movimentos de luta pela democratização de serviços essenciais e de proteção de classe. Entendendo-as como resultantes de um processo político, sua constituição não pode ser pensada como uma elaboração neutra, vazia de ideologia.

Dessa forma, é importante ressaltar que, assim como há diferentes concepções de ciência que norteiam distintas epistemologias, teorias e metodologias, há valores e convicções que direcionam a construção de planos e programas, e o mesmo acontece com as avaliações que buscam compreender seus resultados, geralmente pautados em preceitos economicistas.

Neste estudo, buscou-se situar as especificidades da avaliação de políticas educacionais, destacando a importância de que a análise dos resultados das ações estatais seja construída por uma abordagem plural, formativa e critica. Para isso, defendeu-se que as avaliações precisam se estabelecer como significativas para os sujeitos que utilizam determinado serviço público, superando formatos pautados na tradição de quantificação, como Ball (2011) argumenta, e debruçando-se sobre a compreensão de como a vida dos cidadãos é efetivamente alterada por uma política. Considerou-se isso possivel, todavia, somente com a utilização de uma perspectiva qualitativa, mesmo que esta denote em seu enredamento dificuldades na constituição de um rigor que the dê validação. Contudo, com a concepção de métodos e conceitos alinhados às especificidades dos contextos sociais e à dimensão interpretativa das reflexões dos sujeitos, a validade de tais estudos torna-se crescente no contexto atual.

É nesse sentido que se buscou apontar o ciclo de políticas como uma possibilidade de se realizar avaliações de políticas educacionais engajadas socialmente, que busquem efetivamente perceber como as desigualdades educacionais e sociais são impactadas por determinada política por um viés qualitativo. Entende-se que a especificidade constante nesse modelo o torna 
menos objetivo, o que, em avaliações que envolvam muitos espaços e diferentes sujeitos, pode tornar essa dinâmica mais complexa. No entanto, tal estratégia de avaliação se evidencia como mais propícia à compreensão das singularidades de uma politica e de suas implicações, tornando essas construções mais potentes para a tomada de decisões tanto em relação aos efeitos reais de determinada política quanto acerca da necessidade de implantação de novas políticas.

\section{Referências}

ANDRÉ, Marli. Pesquisa em educação: buscando rigor e qualidade. Cadernos de Pesquisa, n. 113, p. 51-64, 2001. https://doi.org/10.1590/S0100-15742001000200003

ARRETCHE, Marta T. S. Tendências no estudo sobre avaliação de políticas públicas. Terceiro Milênio: Revista Crítica de Sociologia e Política, v. 1, n. 1, p. 128-133, 2013. Disponivel em: http://www.revistaterceiromilenio. uenf.br/index.php/rtm/article/view/64. Acesso em: 4 dez. 2019

BALL, Stephen J. Intelectuais ou técnicos? O papel indispensável da teoria nos estudos educacionais. In: BALL, Stephen J; MAINARDES, Jefferson. (Orgs.). Políticas educacionais: questões e dilemas. São Paulo: Cortez, 2011.

FÁVERO, Altair Alberto; TONIETO, Carina. O lugar da teoria na pesquisa sobre a docência na educação superior. In: _____ (Orgs.). Epistemologias da docência universitária. Curitiba: CRV, 2016.

JAIME, Fernando Martín; DUFOUR, Gustavo; ALESSANDRO, Martín; AMAYA, Paula. Introducción al análisis de politicas públicas. Buenos Aires: Florencio Varela - Universidad Nacional Arturo Jauretche, 2013. Disponivel em: https://www.unaj.edu.ar/wp-content/ uploads/2017/02/Politicas-públicas2013.pdf. Acesso em: 29 nov. 2019.

MAINARDES, Jefferson. Abordagem do ciclo de politicas: uma contribuição para a análise de políticas educacionais. In: Educação \& Sociedade, v. 27, n. 94, p. 47-69, Campinas, 2006. Disponivel em: https://www.scielo.br/ pdf/es/v27n94/a03v27ng4.pdf. Acesso em: 11 dez. 2019.

MAINARDES, Jefferson; FERREIRA, Márcia dos Santos; TELLO, César. Análise de políticas: fundamentos e principais debates teórico-metodológicos. In: BALL, Stephen J; MAINARDES, Jefferson. (Orgs.). Politicas educacionais: questões e dilemas. São Paulo: Cortez, 2011.

NOGUEIRA, Cláudio Marques Martins; NOGUEIRA, Maria Alice. A sociologia da educação de Pierre Bourdieu: limites e contribuições. In: Educação \& Sociedade, ano XXIII, n. 78, p. 15-36, 2002. Disponivel em: https:// www.scielo.br/pdf/es/v23n78/a03v2378.pdf. Acesso em: 10 dez. 2019.
SANDÍN ESTEBAN, Maria Paz. Pesquisa qualitativa em educação: fundamentos e tradições. Porto Alegre: AMGH, 2010.

SANTOS, Boaventura de Sousa. Um discurso sobre as ciências. $5^{\text {a }}$ Ed. São Paulo: Cortez, 2008.

\section{Simone Zanatta Guerra}

Mestrado em andamento em Educação pela Universidade de Passo Fundo (UPF), Brasil. Bolsista da Coordenação de Aperfeiçoamento de Pessoal de Nivel Superior (CAPES). Assistente Social no município de Tapejara (RS).

\section{Renata Cecilia Estormovski}

Mestrado em andamento em Educação pela Universidade de Passo Fundo (UPF), Brasil. Bolsista da Coordenação de Aperfeiçoamento de Pessoal de Nivel Superior (CAPES). Professora e coordenadora pedagógica da rede pública estadual do Rio Grande do Sul.

\section{Endereço para correspondência}

Simone Zanatta Guerra

Rua Independência, 1.066

Centro, 99950000

Tapejara, RS, Brasil

Os textos deste artigo foram revisados pela Zeppelini Publishers e submetidos para validação do(s) autor(es) antes da publicação. 